\title{
Fluorine-18-Fluorodeoxyglucose Positron Emission Tomography in Assessing Retroperitoneal Fibrosis: A Literature Review
}

\author{
Giorgio Treglia, ${ }^{1}$ Maria Vittoria Mattoli, ${ }^{1}$ and Francesco Bertagna ${ }^{2}$ \\ ${ }^{1}$ Institute of Nuclear Medicine, Catholic University of the Sacred Heart, Largo Gemelli, 00168 Rome, Italy \\ ${ }^{2}$ Department of Nuclear Medicine, University of Brescia, Brescia, Italy \\ Correspondence should be addressed to Giorgio Treglia, giorgiomednuc@libero.it
}

Received 29 June 2012; Revised 26 August 2012; Accepted 30 August 2012

Academic Editor: Laszlo Galuska

Copyright (C) 2012 Giorgio Treglia et al. This is an open access article distributed under the Creative Commons Attribution License, which permits unrestricted use, distribution, and reproduction in any medium, provided the original work is properly cited.

\begin{abstract}
Background and Purpose. Several studies have evaluated the role of fluorine-18-fluorodeoxyglucose positron emission tomography and positron emission tomography/computed tomography (FDG-PET and PET/CT) in diagnosing and assessing disease activity in patients with retroperitoneal fibrosis (RF). The aim of our paper is to perform a literature review on this topic. Methods. Scientific articles that evaluated the usefulness of FDG-PET and PET/CT in patients with RF were searched and discussed. Results. Eleven studies were found, and the main findings of these articles were described. Conclusion. FDG-PET and PET/CT are useful functional imaging methods for assessing patients with RF both in the diagnosis and in the treatment response evaluation. Moreover, further studies are needed to substantiate the role of FDG-PET and PET/CT in patients with RF.
\end{abstract}

\section{Introduction}

Retroperitoneal fibrosis (RF) is a chronic inflammatory disease, characterized by the presence of retroperitoneal inflammatory tissue, typically surrounding abdominal aorta and/or iliac arteries, and often leading to the involvement of adjacent structures, more frequently the ureters and inferior vena cava [1-7].

RF is a complex clinical entity still incompletely defined and with unclear etiology. Idiopathic RF (even reported as Ormond's disease) represents two thirds of all cases of RF. A true idiopathic form is present in any cases of RF in which no potential etiologic condition may be identified. The pathogenesis of the idiopathic RF appears today to be related to IgG4 autoimmune mechanisms "hyper-IgG4 disease". Otherwise, RF in the presence of aortic atheromatous inflammation (atheromatous aortitis) has been included, more than twenty years ago, among the secondary forms, since this condition appears to be elicited by antigen-acting oxidizedLDL and/or ceroid, that are present within the atheromatous plaque. Etiology of other secondary RF refers to medications (drug-induced RF), infections, traumas, surgery, radiation therapy, and malignancies [1-7].
Clinical presentation of RF is usually characterized by constitutional symptoms and back or abdominal pain. Because of the presence of increased serum inflammatory markers levels and positive autoantibodies, and the frequent association with autoimmune diseases (such as Riedel's thyroiditis, sarcoidosis, inflammatory aneurysm, and autoimmune pancreatitis), some authors suspected that RF may result from autoimmune mechanisms [7] or, even, be considered as distinct autoimmune diseases $[3,8-11]$.

A frequent complication of RF is unilateral or bilateral ureteral obstruction, secondary to fibrosis and stenosis of the ureters $[1,4,5]$. Other described complications are small and large bowel obstruction and biliary obstruction [12].

The treatment of RF can be medical and/or surgical although a consensus has not been currently reached. The medical therapy is usually based on corticosteroids and immunosuppressive agents (such as tamoxifen) at different doses and various combinations on the base of disease activity and often leads to a substantial reduction of size of the retroperitoneal mass. The surgical procedure is required when severe obstructive complications are present $[4,5,13-$ $15]$. 
However, determining the presence of active inflammation, and thus the potential for response to medical therapy, is crucial for the management of patients with RF and can be quite difficult, especially on the basis of only clinical parameters.

Most commonly used imaging methods in this pathologic entity are computed tomography (CT) and magnetic resonance imaging (MRI), despite their difficulty in discriminating between active and fibrotic lesions and in differentiating between RF and other retroperitoneal diseases such as tumors $[16,17]$.

Fluorine-18-fluorodeoxyglucose positron emission tomography and positron emission tomography/computed tomography (FDG-PET and PET/CT) are noninvasive functional imaging techniques which have become established tools in oncology [18]. Recently, FDG-PET and PET/CT have been proposed as potentially useful tools for study inflammatory diseases, such as large-vessel vasculitis (LVV) and atherosclerosis [19-23]. In fact, FDG is a glucose analogue that identifies areas of high-glucose metabolism. Since inflammatory cells, such as neutrophils, lymphocytes, and macrophages, have an increased glucose metabolism, high FDG uptake is revealed in vessel walls and retroperitoneal masses if inflammation is present [19-23].

Several studies have evaluated the role of FDG-PET and $\mathrm{PET} / \mathrm{CT}$ in diagnosing, assessing disease activity, and monitoring RF. The aim of our paper is to review relevant published articles on this topic.

\section{Literature Data about FDG-PET and PET/CT in Patients with Retroperitoneal Fibrosis}

A comprehensive computer literature search of PubMed/ MEDLINE was conducted in order to find relevant published articles on the role of FDG-PET and PET/CT in patients with RF. We used a search algorithm based on a combination of the following terms: (a) "retroperitoneal fibrosis" or "Ormond" and (b) "positron emission tomography" or "PET." No beginning date limit was used; the search was updated until July 31st 2012. Studies investigating the role of FDG-PET and PET/CT in patients with RF were eligible for inclusion. Review articles or editorials, articles not in the field of interest of this paper, case reports, and preclinical studies were excluded.

Eleven relevant articles about the role of FDG-PET and PET/CT in patients with RF were found and discussed (Table 1).

In 2005, Salvarani et al. [24] evaluated the presence and extent of large-vessel inflammation in 7 patients with RF or other chronic periaortitis using FDG-PET. These authors found FDG-PET findings suggestive for LVV in patients with RF, demonstrating increased vascular radiopharmaceutical uptake involving abdominal aorta and/or common iliac arteries, which in some cases was also extended to the thoracic aorta and/or its branches [24].

In the same year, Vaglio et al. [25] used FDG-PET to evaluate the metabolic activity of residual masses at CT scan after immunosuppressive treatment in patients with RF. Six out of seven patients evaluated had no abnormal FDG uptake in the residual mass, and the seventh showed only moderate vascular FDG uptake. During the followup, none of the patients showed clinical, serological, or radiological signs of disease relapse. These findings suggested that FDG-PET is a sensitive mean to evaluate metabolic activity of residual masses in RF patients in clinical remission with detectable masses at CT scans [25].

In 2007, Nakajo et al. [26] reviewed six male patients with $\mathrm{RF}$ and confirmed that FDG-PET and PET/CT are useful tools for evaluating disease activity and extent of RF although these methods failed to differentiate between malignancy and RF. In fact, the authors performed early and delayed dualtime point imaging to examine the change of FDG uptake in RF, finding an increase of maximum standardized uptake value (SUVmax) at delayed images, which was similar to the pattern observed in malignancies. Abnormal FDG uptake was also noted in the mediastinum and in the pancreas of some patients, and the diagnosis of mediastinal fibrosis and autoimmune pancreatitis were made, respectively [26].

In 2008, Young et al. [27] reported FDG-PET-positive findings in three patients presenting with active RF. In one patient, repeat FDG-PET was performed following immunosuppressive therapy, with complete resolution of the retroperitoneal FDG uptake. The authors confirmed that FDGPET may be a useful adjunct to anatomic imaging and serum inflammatory markers in distinguishing between active and inactive disease and in assessing the severity of inflammation in RF. This method may also be used in followup to assess therapeutic response if CT findings are unclear [27].

In 2010, Jansen et al. [28] evaluated whether FDG-PET was useful in the diagnostic and therapeutic evaluation of patients with RF treated with tamoxifen. At baseline, FDGPET was positive in $20 / 26$ patients. Patients with a positive FDG-PET scan had a higher serum inflammatory markers level $(P=0.02)$ and a larger mass size at CT scan $(P=0.01)$ compared with patients with a negative FDG-PET scan. FDG uptake decreased following treatment, in agreement with inflammatory markers reduction $(P<0.001)$, but not with CT-documented mass regression. These findings confirmed that FDG-PET may be useful to evaluate the severity and the extent of RF. Furthermore, these authors demonstrated that FDG-PET may be a valuable tool in assessing disease activity during or after treatment in patients with normal inflammatory marker levels and stable residual mass on repeated CT scans. Nevertheless, short-term FDG-PET followup did not accurately predict success of tamoxifen treatment [28].

In the same year, Piccoli et al. [29] supported the use of FDG-PET/CT not only for the diagnostic workup but also in the followup of patients with RF in order to tailor medical and surgical approaches. In particular, evaluating seven RF patients with FDG-PET/CT during the diagnostic workup and during followup, the authors found that this method may be useful to identify the best time for a safe removal of the renal stents (when no active disease is revealed by FDGPET). FDG-PET/CT has been also useful to schedule for tapering of immunosuppressive therapy [29]. 
TABLE 1: Characteristics of the included articles.

\begin{tabular}{|c|c|c|c|c|c|c|c|}
\hline Authors & Year & Country & $\begin{array}{l}\text { Type of } \\
\text { study }\end{array}$ & $\begin{array}{c}\text { Patients } \\
\text { evaluated by } \\
\text { FDG-PET }\end{array}$ & Device used & $\begin{array}{l}\text { Analysis of PET } \\
\text { images }\end{array}$ & PET timing \\
\hline Salvarani et al. [24] & 2005 & Italy & Retrospective & 5 IRF, 2 PRF & PET & Visual & Before treatment \\
\hline Vaglio et al. [25] & 2005 & Italy & Retrospective & 7 IRF & PET & Visual & After treatment \\
\hline Nakajo et al. [26] & 2007 & Japan & Retrospective & $6 \mathrm{IRF}$ & $\begin{array}{l}\text { PET and } \\
\mathrm{PET} / \mathrm{CT}\end{array}$ & $\begin{array}{l}\text { Visual and } \\
\text { semi-quantitative }\end{array}$ & $\begin{array}{l}\text { Before treatment in } 5 \\
\text { patients and after treatment } \\
\text { in } 1 \text { patient }\end{array}$ \\
\hline Young et al. [27] & 2008 & USA & Retrospective & 3 IRF & PET & $\begin{array}{l}\text { Visual and semi } \\
\text { quantitative }\end{array}$ & $\begin{array}{l}\text { Before treatment in } 3 \\
\text { patients and after treatment } \\
\text { in } 1 \text { patient }\end{array}$ \\
\hline Jansen et al. [28] & 2010 & $\begin{array}{l}\text { The } \\
\text { Netherlands }\end{array}$ & Prospective & 26 IRF & PET & Visual & $\begin{array}{l}\text { Before treatment in } 26 \\
\text { patients and after treatment } \\
\text { in } 18 \text { patients }\end{array}$ \\
\hline Piccoli et al. [29] & 2010 & Italy & Prospective & 7 IRF & $\mathrm{PET} / \mathrm{CT}$ & $\begin{array}{l}\text { Visual and semi } \\
\text { quantitative }\end{array}$ & Before and after treatment \\
\hline Pipitone et al. [30] & 2011 & Italy & Retrospective & 1 IRF; 3 SRF & PET & Visual & Before and after treatment \\
\hline Ha et al. [31] & 2011 & Korea & Retrospective & $6 \mathrm{IRF}$ & PET & Visual & Before treatment \\
\hline Bertagna et al. [32] & 2012 & Italy & Retrospective & $25 \mathrm{IRF}$ & $\mathrm{PET} / \mathrm{CT}$ & $\begin{array}{l}\text { Visual and semi } \\
\text { quantitative }\end{array}$ & $\begin{array}{l}\text { Before treatment in } 18 \\
\text { patients and after treatment } \\
\text { in } 17 \text { patients }\end{array}$ \\
\hline Guignard et al. [33] & 2012 & Switzerland & Retrospective & 7 IRF, 3 SRF & $\mathrm{PET} / \mathrm{CT}$ & $\begin{array}{l}\text { Visual and semi } \\
\text { quantitative }\end{array}$ & Before and after treatment \\
\hline Moroni et al. [34] & 2012 & Italy & Prospective & $22 \mathrm{IRF}$ & $\mathrm{PET} / \mathrm{CT}$ & $\begin{array}{l}\text { Visual and semi } \\
\text { quantitative }\end{array}$ & Before and after treatment \\
\hline
\end{tabular}

Legend: IRF: idiopathic retroperitoneal fibrosis, SRF: secondary retroperitoneal fibrosis; PRF: perianeurysmal retroperitoneal fibrosis.

Recently, Pipitone et al. [30] presented four cases of RF with different etiology (one idiopathic RF and three secondary RF) and evaluated the role of FDG-PET in the workup of these different conditions. The authors highlighted that FDG-PET should be part of the investigations used to determine disease extent, activity, and response to therapy both in patients with idiopathic and secondary RF [30].

$\mathrm{Ha}$ et al. [31] recently investigated the clinical characteristics, laboratory findings, radiologic findings, treatment, and outcome in 27 Korean patients with RF. Only 6/27 patients have performed FDG-PET scan, and five out of six patients showed FDG-PET-positive findings. The authors suggested that FDG-PET may be a useful tool to detect active $\mathrm{RF}[31]$.

Bertagna et al. [32] assessed the feasibility and usefulness of FDG-PET/CT in $25 \mathrm{RF}$ patients studied in two Italian nuclear medicine centres; 18/25 patients underwent PET/CT as initial evaluation study, $3 / 25$ patients during followup, $3 / 25$ during steroid therapy in absence of initial evaluation, and $1 / 25$ to reevaluate the disease suspecting a recurrence. FDG-PET/CT was positive in $18 / 25$ patients and negative in $7 / 25$. Among the 10 patients who underwent a second study after steroid therapy, 6/10 showed complete metabolic response, 3/10 partial response, and one patient showed no significant FDG uptake reduction. The authors concluded that FDG-PET/CT is a suitable and useful tool in staging patients which are suspected to be affected by RF; moreover it could be very useful in treatment response evaluation [32].
Guignard et al. [33] evaluated the added value of contrast-enhanced CT (CE-CT) combined with FDG-PET as a one-stop diagnostic procedure for the assessment and followup of RF in 10 patients. These authors found that, unlike biologic and CT parameters, FDG uptake, was the most relevant parameter to measure severity of inflammation in RF. CE-CT allowed a better delineation of periaortitis and its extension to adjacent structures. The authors concluded that accurate determination of inflammation level in $\mathrm{RF}$ is significantly improved when FDG-PET/CT and CE-CT are performed in the same study and used for better delineation of areas of residual inflammation [33].

Lastly, Moroni et al. [34] in their recent prospective study on 22 patients with RF reported that FDG-PET/CT accurately discriminated active from inactive disease in about $94 \%$ of cases. A significant correlation $(P<0.01)$ was found among FDG uptake, and serum levels of inflammatory markers and among FDG uptake and analysis of contrast enhancement at CT images in the retroperitoneal mass. The authors concluded that FDG PET/CT may be considered an alternative imaging method for the assessment of different stages of RF [34].

\section{General Remarks about FDG-PET and PET/CT in Patients with Retroperitoneal Fibrosis}

The diagnosis of RF is based on clinical and radiological findings, and there are no established PET criteria for the 
diagnosis of this pathological entity. Many authors used a visual analysis of PET images for the evaluation of metabolic activity of RF. The visual analysis was usually performed using a four-point graded scale, based on the vessel-to-liver FDG uptake ratio (0: no uptake, 1: uptake less than that of the liver, 2: uptake equal to that of the liver, and 3: uptake greater than that of the liver); a visual score above 1 was usually considered a positive criteria for active RF.

However, standardization of FDG-PET studies by using semiquantitative analysis in order to achieve interchangeability in multicenter trials was recently recommended [35]. Six out of 11 articles included in this review have used a semiquantitative analysis based on the calculation of the SUVmax $[26,27,29,32-34]$. The semiquantitative assessment of FDG uptake in the RF lesions is also useful for treatment response evaluation.

The added value of the hybrid technique PET/CT versus PET alone for RF diagnosis needs to be confirmed by further studies. In fact, only four articles evaluated the usefulness of FDG-PET/CT in patients with RF [29, 32-34]. Hybrid $\mathrm{PET} / \mathrm{CT}$ allows the evaluation of disease activity and retroperitoneal mass size, as well as the localization of the inflammatory process in the same session. The main limitation of CT scan is the lack of discrimination between active and residual fibrotic tissue; nevertheless, CT may be useful in differentiating RF from atherosclerosis or in detecting morphological vascular alterations such as stenoses or aneurysms. Furthermore, a recent study suggested that the combination of FDG-PET and CE-CT performed in a single procedure would simplify the patient management [33]. This strategy could be more cost-effective and more accurate than separate studies acquired at different points in time although randomized prospective studies are required to validate this hypothesis [33].

Overall, FDG-PET and PET/CT seem to be useful methods both in evaluating disease extent and severity at the time of diagnosis and in assessing disease activity during or after treatment, mainly in patients with normal inflammatory marker levels and stable residual mass on repeated CT scans. Further prospective and multicentric studies with a larger patient population and cost-effectiveness studies are needed to correctly define the role of FDG-PET and PET/CT in patients with RF.

\section{Conclusions}

From this overview on the role of FDG-PET and PET/CT in patients with RF, we are led to conclude that these functional imaging techniques seem to be useful both in the diagnosis and in evaluating the treatment response. Further studies are needed to substantiate the role of FDG-PET and PET/CT in this setting.

\section{Conflict of Interests}

The authors declare that they have no conflict of interests.

\section{References}

[1] A. Vaglio, C. Salvarani, and C. Buzio, "Retroperitoneal fibrosis," The Lancet, vol. 367, no. 9506, pp. 241-251, 2006.

[2] C. Alberti, "Retroperitoneal fibroses: aetiopathogenesis and taxonomic assessment," European Review for Medical and Pharmacological Sciences, vol. 11, no. 6, pp. 375-382, 2007.

[3] E. S. Haug, J. F. Skomsvoll, G. Jacobsen, T. B. Halvorsen, O. D. Saether, and H. O. Myhre, "Inflammatory aortic aneurysm is associated with increased incidence of autoimmune disease," Journal of Vascular Surgery, vol. 38, no. 3, pp. 492-497, 2003.

[4] N. Pipitone, C. Salvarani, and H. H. Peter, "Chronic periaortitis," Internist, vol. 51, no. 1, pp. 45-52, 2010.

[5] A. Palmisano and A. Vaglio, "Chronic periaortitis: a fibroinflammatory disorder," Best Practice and Research, vol. 23, no. 3, pp. 339-353, 2009.

[6] D. Hughes and P. J. Buckley, "Idiopathic retroperitoneal fibrosis is a macrophage-rich process: implications for its pathogenesis and treatment," American Journal of Surgical Pathology, vol. 17, no. 5, pp. 482-490, 1993.

[7] A. Vaglio, P. Greco, D. Corradi et al., "Autoimmune aspects of chronic periaortitis," Autoimmunity Reviews, vol. 5, no. 7, pp. 458-464, 2006.

[8] A. Vaglio, D. Corradi, L. Manenti, S. Ferretti, G. Garini, and C. Buzio, "Evidence of autoimmunity in chronic periaortitis: a prospective study," American Journal of Medicine, vol. 114, no. 6, pp. 454-462, 2003.

[9] G. O. Littlejohn and E. C. Keystone, "The association of retroperitoneal fibrosis with systemic vasculitis and HLA-B27: a case report and review of the literature," Journal of Rheumatology, vol. 8, no. 4, pp. 665-669, 1981.

[10] H. Okada, S. Takahira, S. Sugahara, H. Nakamoto, and H. Suzuki, "Retroperitoneal fibrosis and systemic lupus erythematosus," Nephrology Dialysis Transplantation, vol. 14, no. 5, pp. 1300-1302, 1999.

[11] G. Moroni, A. Farricciotti, M. Cappelletti, and C. Ponticelli, "Retroperitoneal fibrosis and membranous nephropathy. Improvement of both diseases after treatment with steroids and immunosuppressive agents," Nephrology Dialysis Transplantation, vol. 14, no. 5, pp. 1303-1305, 1999.

[12] A. S. Brandt, L. Kamper, S. Kukuk, P. Haage, and S. Roth, "Associated findings and complications of retroperitoneal fibrosis in 204 patients: results of a urological registry," Journal of Urology, vol. 185, no. 2, pp. 526-531, 2011.

[13] A. Vaglio, "Retroperitoneal fibrosis: new insights into clinical presentation and diagnosis," Medicine, vol. 88, no. 4, pp. 208$210,2009$.

[14] N. Pipitone, C. Salvarani, and H. H. Peter, "Chronic periaortitis," Internist, vol. 51, no. 1, pp. 45-52, 2010.

[15] A. Palmisano and A. Vaglio, "Chronic periaortitis: a fibroinflammatory disorder," Best Practice and Research, vol. 23, no. 3, pp. 339-353, 2009.

[16] I. Vivas, A. I. Nicolás, P. Velázquez, B. Elduayen, T. FernándezVilla, and A. Martínez-Cuesta, "Retroperitoneal fibrosis: typical and atypical manifestations," British Journal of Radiology, vol. 73, no. 866, pp. 214-222, 2000.

[17] C. G. Cronin, D. G. Lohan, M. A. Blake, C. Roche, P. McCarthy, and J. M. Murphy, "Retroperitoneal fibrosis: a review of clinical features and imaging findings," American Journal of Roentgenology, vol. 191, no. 2, pp. 423-431, 2008.

[18] G. Treglia, E. Cason, and G. Fagioli, "Recent applications of nuclear medicine in diagnostics (I part)," Italian Journal of Medicine, vol. 4, no. 2, pp. 84-91, 2010. 
[19] G. Treglia, E. Cason, and G. Fagioli, "Recent applications of nuclear medicine in diagnostics: II part," Italian Journal of Medicine, vol. 4, pp. 159-166, 2010.

[20] M. Gotthardt, C. P. Bleeker-Rovers, O. C. Boerman, and W. J. G. Oyen, "Imaging of inflammation by PET, conventional scintigraphy, and other imaging techniques," Journal of Nuclear Medicine, vol. 51, no. 12, pp. 1937-1949, 2010.

[21] G. Treglia, M. V. Mattoli, L. Leccisotti, G. Ferraccioli, and A. Giordano, "Usefulness of whole-body fluorine-18-fluorodeoxyglucose positron emission tomography in patients with large-vessel vasculitis: a systematic review," Clinical Rheumatology, vol. 30, pp. 1265-1275, 2011.

[22] N. Tahara, T. Imaizumi, R. Virmani, and J. Narula, "Clinical feasibility of molecular imaging of plaque inflammation in atherosclerosis," Journal of Nuclear Medicine, vol. 50, no. 3, pp. 331-334, 2009.

[23] M. V. Mattoli, G. Treglia, L. Leccisotti, and A. Giordano, "The role of ${ }^{18} \mathrm{~F}-\mathrm{FDG}$ PET/CT in the management of large-vessel vasculitis: applications and limitations in clinical practice," Italian Journal of Medicine, vol. 5, pp. 249-254, 2011.

[24] C. Salvarani, N. Pipitone, A. Versari et al., "Positron emission tomography (PET): evaluation of chronic periaortitis," Arthritis Care \& Research, vol. 53, no. 2, pp. 298-303, 2005.

[25] A. Vaglio, P. Greco, A. Versari et al., "Post-treatment residual tissue in idiopathic retroperitoneal fibrosis: active residual disease or silent "scar"? A study using 18F-fluorodeoxyglucose positron emission tomography," Clinical and Experimental Rheumatology, vol. 23, no. 2, pp. 231-234, 2005.

[26] M. Nakajo, S. Jinnouchi, H. Tanabe, R. Tateno, and M. Nakajo, "18 F-fluorodeoxyglucose positron emission tomography features of idiopathic retroperitoneal fibrosis," Journal of Computer Assisted Tomography, vol. 31, no. 4, pp. 539-543, 2007.

[27] P. M. Young, J. J. Peterson, and K. T. Calamia, "Hypermetabolic activity in patients with active retroperitoneal fibrosis on F-18 FDG PET: report of three cases," Annals of Nuclear Medicine, vol. 22, no. 1, pp. 87-92, 2008.

[28] I. Jansen, T. R. Hendriksz, S. H. Han, A. W. L. C. Huiskes, and E. F. H. van Bommel, "18F-fluorodeoxyglucose position emission tomography (FDG-PET) for monitoring disease activity and treatment response in idiopathic retroperitoneal fibrosis," European Journal of Internal Medicine, vol. 21, no. 3, pp. 216-221, 2010.

[29] G. B. Piccoli, V. Consiglio, V. Arena et al., "Positron emission tomography as a tool for the 'tailored' management of retroperitoneal fibrosis: a nephro-urological experience," Nephrology Dialysis Transplantation, vol. 25, no. 8, pp. 2603-2610, 2010.

[30] N. Pipitone, A. Versari, A. Vaglio, and C. Salvarani, "Role of ${ }^{18} \mathrm{~F}$-fluorodeoxyglucose positron emission tomography in the workup of retroperitoneal fibrosis," Clinical and Experimental Rheumatology, vol. 29, no. 1, supplement 64, pp. S72-S78, 2011.

[31] Y. J. Ha, S. J. Jung, K. H. Lee, S. W. Lee, S. K. Lee, and Y. B. Park, "Retroperitoneal fibrosis in 27 Korean patients: single center experience," Journal of Korean Medical Science, vol. 26, pp. 985-990, 2011.

[32] F. Bertagna, G. Treglia, L. Leccisotti et al., "[( $\left.{ }^{18}\right)$ F]FDG-PET/ $\mathrm{CT}$ in patients affected by retroperitoneal fibrosis: a bicentric experience," Japanese Journal of Radiology, vol. 30, no. 5, pp. 415-421, 2012.

[33] R. Guignard, M. Simukoniene, V. Garibotto, and O. Ratib, " ${ }^{18}$ F-FDG PET/CT and contrast-enhanced CT in a one-stop diagnostic procedure: a better strategy for management of patients suffering from retroperitoneal fibrosis?" Clinical Nuclear Medicine, vol. 37, pp. 453-459, 2012.

[34] G. Moroni, M. Castellani, A. Balzani et al., "The value of $\left({ }^{18}\right) \mathrm{F}-$ FDG PET/CT in the assessment of active idiopathicretroperitoneal fibrosis," European Journal of Nuclear Medicine and Molecular Imaging. In press.

[35] R. Boellaard, W. J. G. Oyen, C. J. Hoekstra et al., "The Netherlands protocol for standardisation and quantification of FDG whole body PET studies in multi-centre trials," European Journal of Nuclear Medicine and Molecular Imaging, vol. 35, no. 12 , pp. 2320-2333, 2008. 


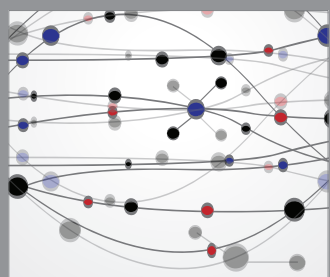

The Scientific World Journal
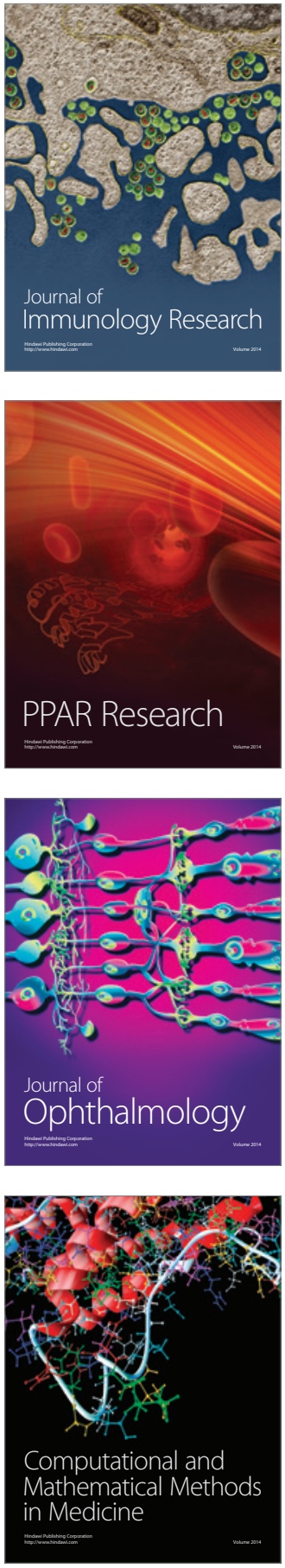

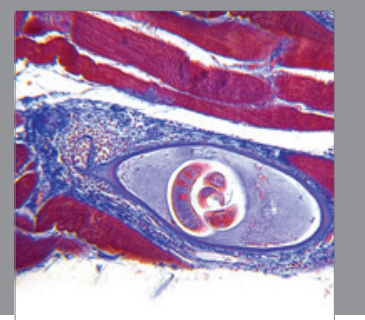

Gastroenterology

Research and Practice
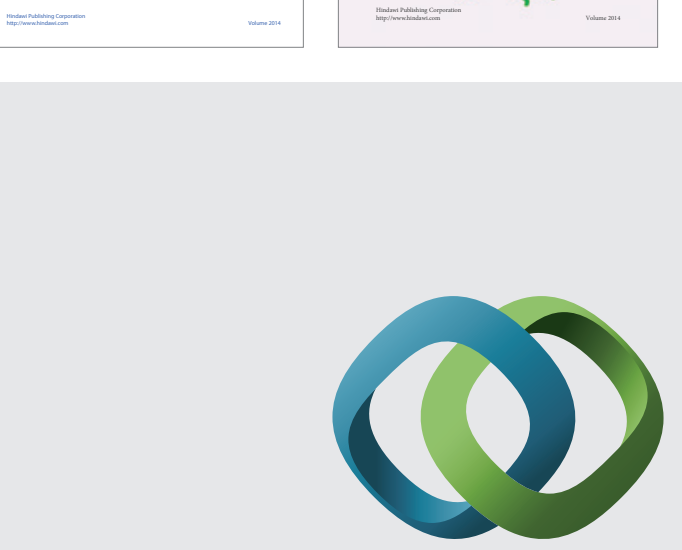

\section{Hindawi}

Submit your manuscripts at

http://www.hindawi.com
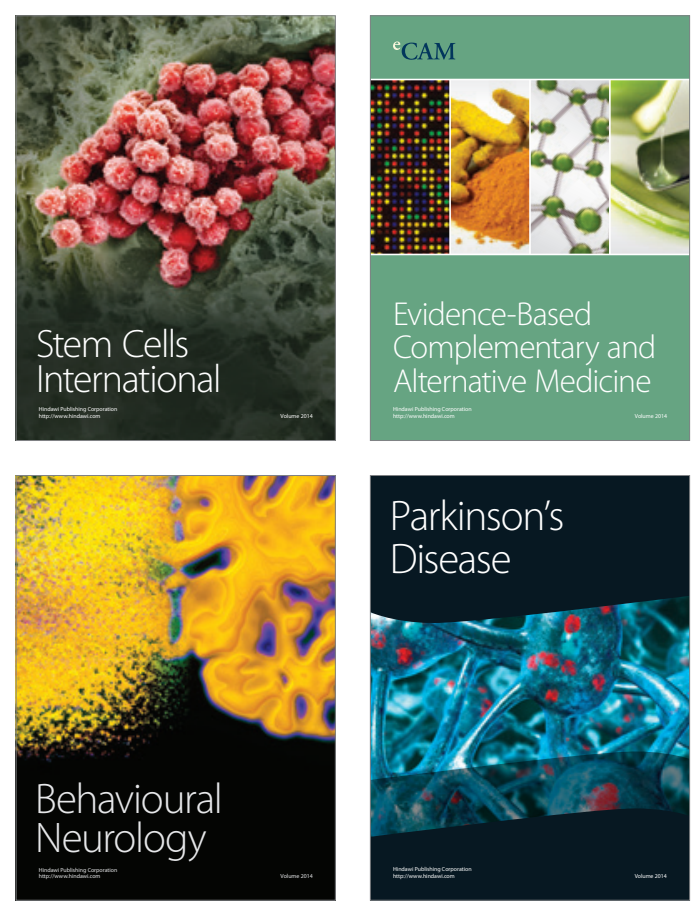

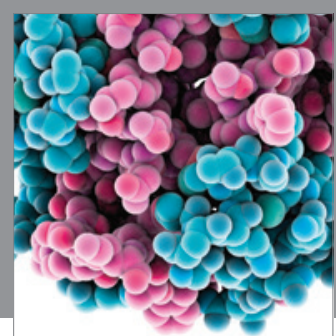

Journal of
Diabetes Research

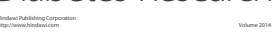

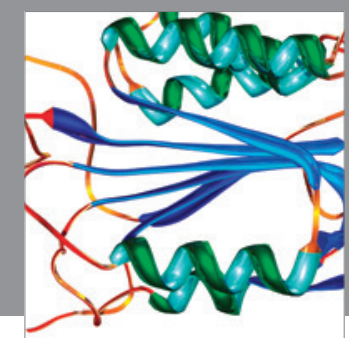

Disease Markers
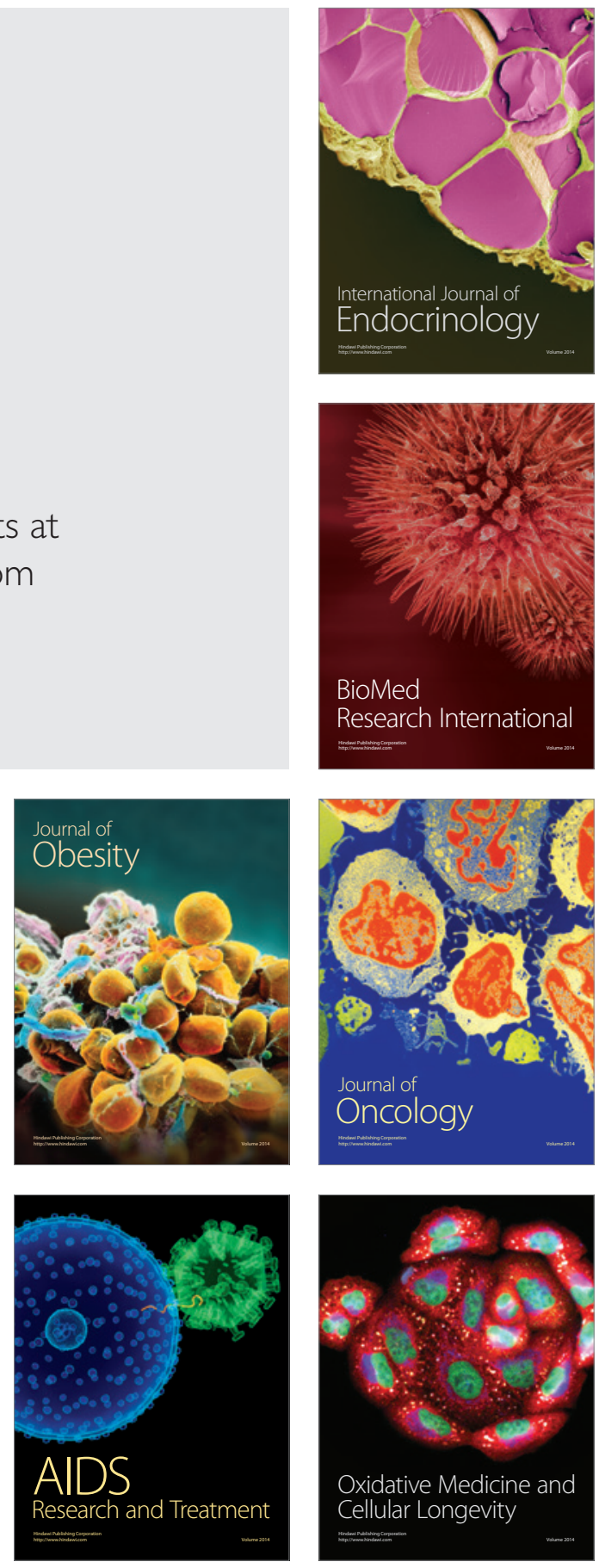